\title{
Self- tuning Fuzzy PID controller on DCIDC Converter based on SA-GA
}

\author{
Jiancheng Zhou, Qingyong Zhang, and Yihao Wan \\ School of Automation, Wuhan University of Technology Wuhan, 430070 China \\ zhoujiancheng@whut.edu.cn
}

\begin{abstract}
Keywords: DC/DC Converter, Simulated Annealing, Genetic Algorithm, Fuzzy PID, MATLAB Simulation
\end{abstract}

\begin{abstract}
Modern DC/DC Converter has characteristics of high efficiency, good output noiseand low voltage ripple. It is wildly used in communication, computer, industry automation,military and aerospace. However, due to its strong nonlinear, time dependent and uncertainsystem, to some extent, conventional PID control method can improve the performance of DC/DCconverters, the parameter turning is rather complex and hard to get the best performance of DC/DC converter. This paper presents a method of combining simulated annealing genetic algorithm and fuzzy PID in DC/DC converter, giving fully play to the robustness andself-adaptive ability of PID, with simultaneous intelligent parameter search. Simulated annealing genetic algorithm overcome the defects of local optimization and premature convergence. The proposed self-optimizing fuzzy PID controller is implemented by simulating BUCK circuit onmatlab. It is shown that the system achieves good self-adaptiveness, fast response, low overshoot and strong robustness.
\end{abstract}

\section{Introduction}

Conventional PID is an effective control method developed early. Especially for the object with precise mathematical model, it can achieve excellent performance. However, it is difficult to build precise models due to strong nonlinear and chaotic property of DC/DC converter. And PID control algorithm may cause overshoot, bad noise immunity and time-consuming parameter turning. Fuzzy PID control algorithm proposed by many literatures, which is independent on specific mathematical model, can effectively suppress overshoot and strengthen robustness. However, in comparison to PID algorithm, it also has defectsof long response timeand poor stability.

This paper combines the advantages of fuzzy algorithm and PID algorithm, proposing a self-optimizing fuzzy PID algorithm based on simulated annealing genetic algorithm(SAGA). Using parallel-search capability of a genetic algorithm, the SA algorithm can avoid local optimization andreach global optimization. The combination greatly improved the solution search ability and reduce the time of search. This algorithm has characteristics of fast response, low overshoot, strong robustness and easy parameter turning. It greatly improves the performanceof DC/DC converters and stability of the system.

\section{Conventional PID controlled BUCK Circuit}

Conventional PID controlled BUCK circuit is shown in Fig.2-1, it send feedback to the closed-loop system through the proportion(P), integration(I) and differentiation(D) of the difference between ideal output and real output, and improves its performance. It is widely used in control systems.

The ideal continuous-time PID controller can be written as:

$$
u(t)=K p\left(e(t)+\frac{1}{T i} \cdot \int e(t) d t+K d \cdot \frac{d e}{d t}\right)(2-1)
$$

Where theproportional gain is $\mathrm{Kp}$, integral gain $\mathrm{Ki}$ is $\mathrm{Kp} / \mathrm{Ti}$, derivative gain $\mathrm{Kd}$ is $\mathrm{Kp} * \mathrm{Td}$. The system model can also be expressed as:

$$
u(t)=K p \cdot e(t)+K i \cdot \int e(t) d t+K d \cdot \frac{d e}{d t}(2-2)
$$


Evidently, it is crucial to adjust the three parameter $\mathrm{Kp}, \mathrm{Ki}, \mathrm{Kd}$ of PID controller.

(1)Kp, proportional gain, have the effect of accelerating the response speed, reduce systematic error, but can also make the system unstable if too large.

(2)Ki, integral gain, have the effect of eliminating the steady-state error and indiscrimination degree, but it may lower the stability of system and the adjusting time is long.

(3)Kd, derivative gain, have the effect of increasing the stability of the system, reducing the overshoot, decreasing the adjusting time, but it may amplify the noise and has poor noise immunity.

As for common control system, adjusting Kp, Ki, Kd can achieve good control performance, but Due to strong nonlinear characteristic of DC/DC converter, the control performance of self-optimizing PID is hard to satisfy the needs. And it is difficult to adjust the three parameters spontaneously. It is determined by cut and try method usually, but the method not only wastes of time, but also difficult to be turned.

The simulation results show that the conventional controlled BUCK circuit has large output voltage and current overshoot. It is unfavorable to increase the frequency of switching power supply and improve the performance.

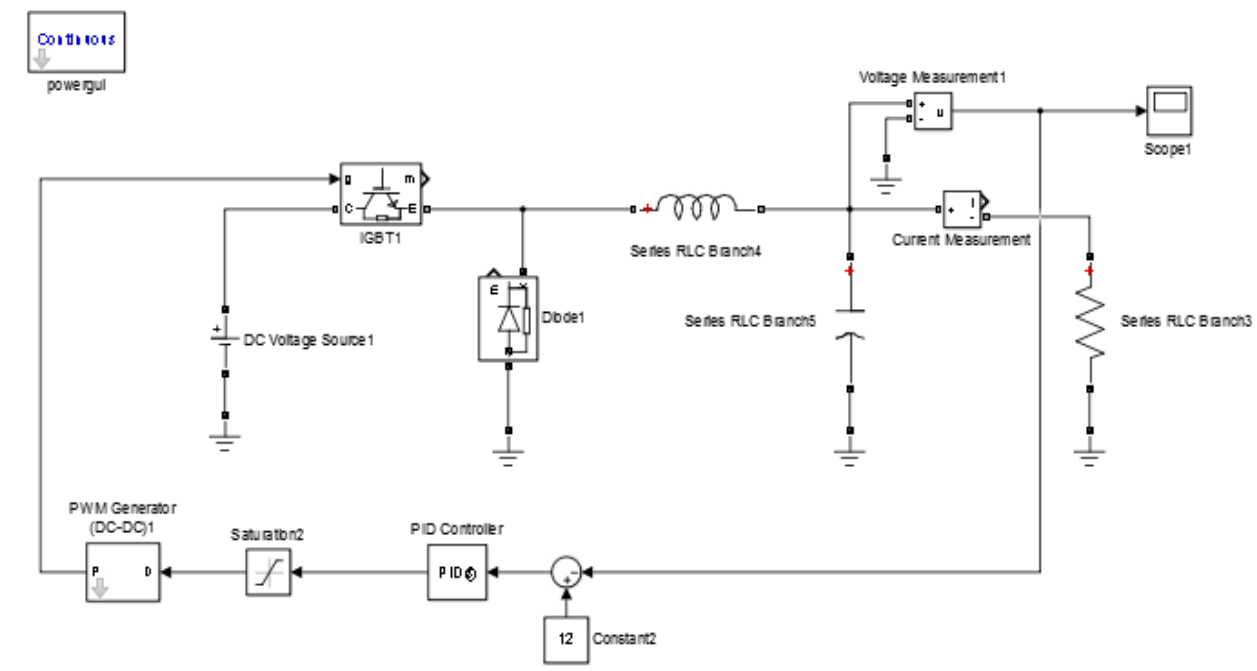

Fig.1 Simulation of PID-BUCK Circuit

\section{The principle of BUCK circuit's control structure}

The system consists of SAGA Parameter Tuning Device, Fuzzy inferior, PID controller and controlled object. SAGA Parameter Tuning Device input the deviation e between input and output, and calculate the fitness based on fitness function. It outputs the quantization factorKe and Kec of fuzzy inferior, and then infers suitable parameters for PID controller. Using the parameters in controlled object to do incessant parameter self-turning. So the system has concentrated fast response of PID and small overshoot of fuzzy control. Moreover, it has strong robustness and can realize global optimization independent to specific function model. The performance of system is improved greatly.

Fig 2 is schematic diagram of system structure. It is co-simulated on the platform of MATLAB with Simulink and S-Function, and the results confirm the successful realization of the system. 


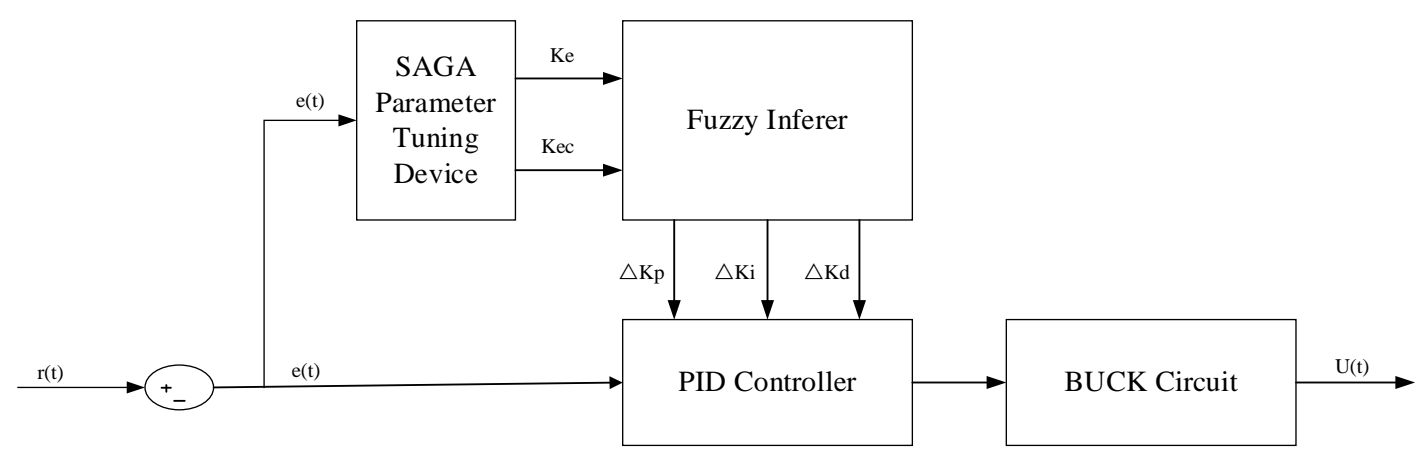

Fig 2 Schematic diagram of BUCK Circuit

\section{The design of controller}

In this paper, the designed controller include fuzzy controller. The fuzzy controller is specially designed to regulate the parameter of PID, to reduce the complexity of turning PID parameter. It input error factor e and the changing rate of error ec. PID parameter regulating factors $\Delta \mathrm{Kp} 、 \Delta \mathrm{Ki}$ 、 $\Delta \mathrm{Kd}$ is obtained by fuzzification, establishing rule library, logical judgment and defuzzification. And we obtain new parameters of PID:

$$
\left\{\begin{array}{l}
K_{p}=K_{p 0}+\Delta K_{p} \\
K_{i}=K_{i 0}+\Delta K_{i} \\
K_{d}=K_{d_{0}}+\Delta K_{d}
\end{array}\right.
$$

So a Fuzzy PID controller is properly designed, there are many fuzzy PID controllers in literatures. In this paper, we quoted the two-input and three-output fuzzy PID controller in literature [4].

Genetic Algorithms(GAs) are parallel search algorithms of probability, based on the principle of natural selection. It can break through the single point search defect of conventional optimizing algorithm and realize overall search. However, the speed of search is limited compared with global search, and it can easily fall into local optimization and premature. Simulated annealing (SA) is a probabilistic technique based on solid annealing. In the process of cooling, simulatedannealing accepts current inferior solution using probability control, to avoid the local optimization defect of common algorithm. This paper combines the advantages of the two algorithm, and introduces annealing operator into Genetic Algorithm to accelerate the convergence, to reach global optimization. The structure of the algorithm is shown in Fig.3, 


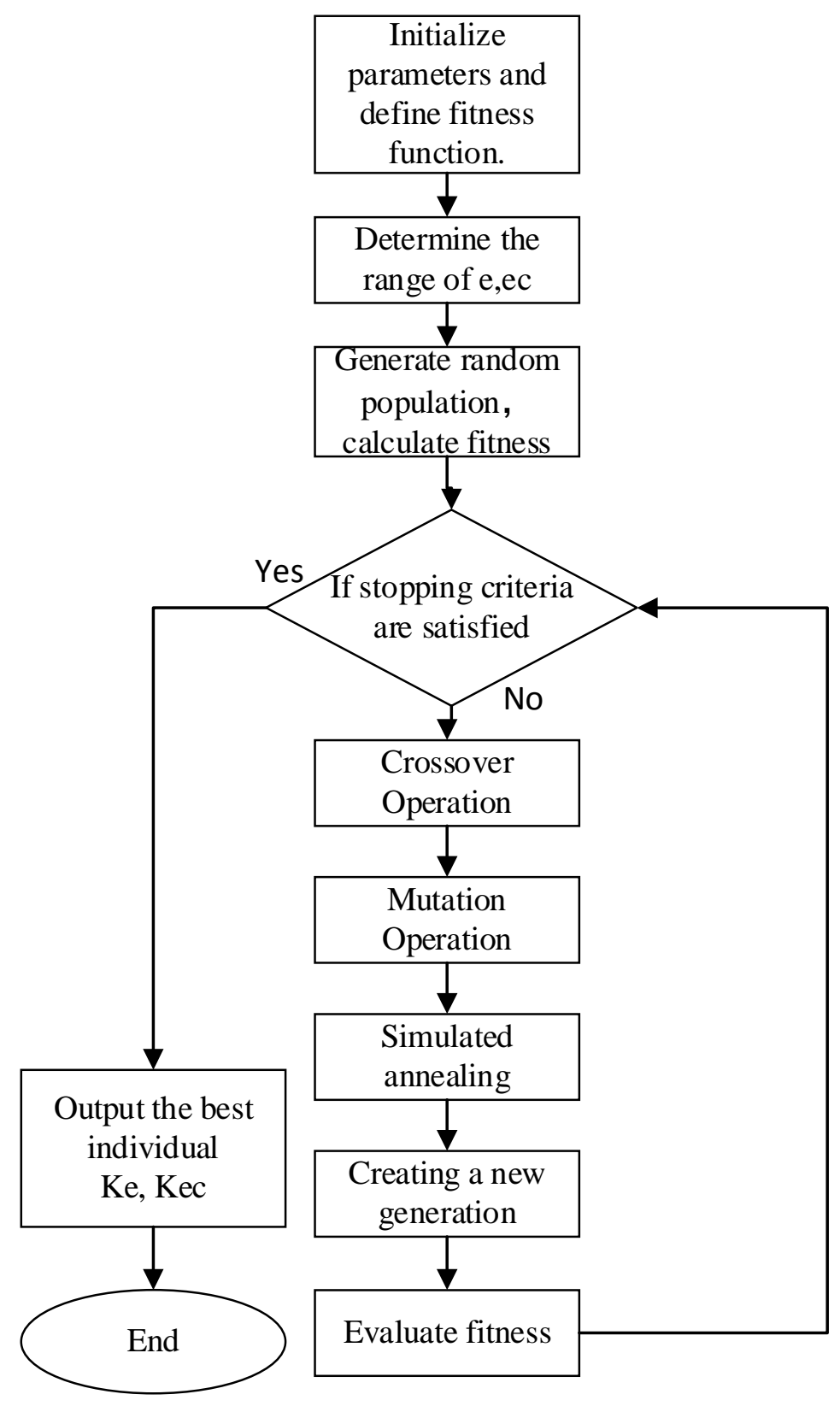

Fig.3 SAGA Algorithm

The SAGA algorithm can be summarized as follows:

Step1: Initialize population, set the population size $\mathrm{n}$, iteration times $\mathrm{T}$, chromosome length $\mathrm{m}$, crossover probability Pc, mutation probability Pm, initial temperature T0, and confirm individual fitness function.

Step2: Confirm the range of Ke and Kec.

Step3: Generate random population, compute the fitness.

Step4: Perform the crossover.

Step5: Perform the mutation.

Step6: Perform simulated annealing, accept current inferior individual on the basis of controlled probability.

Step7: Evaluate the fitness, perform the terminating test. If satisfies the stopping criterion, then output the optimum individual, otherwise, go to step4.

Step8: End

(1) Encode the variants. This algorithm has two variables Ke and Kec, there are many methods to encode, and binary code is the most commonly used method. It is easy and feasible, moreover, it is easy to analyze using schema theorem. So, in this paper, we adopted Binary Code. 
(2) Confirm fitness function. In the process of searching optimization information, GA is independent of external information and is only associated with fitness function. Hence, it is crucial to confirm the fitness function. In the process of stabilization of BUCK circuit, in order to make the system smoothly stabilized, taking the integration of absolute value of error signal as judgment criterion, to maximize the function. The fitness function is

$$
f=\frac{1}{\int|e| d t}
$$

(3) To get better approximate solutions, perform crossover and recombination to population based on controlled crossover probability Pc and mutation probability Pm. And mutate the individuals, the offspring approaches optimal solution after certain iteration times.

(4) Simulated annealing operation. Due to the size limit of genetic search, it is easy to get stuck in local optimization and cannot achieve the goal of global optimization. The searching cycle is time-consuming, it cannot end search in advance. Thus, we introduce a parameter $\alpha$, decreasing to Tt in iteration:

$$
T t=T t \cdot \alpha
$$

In the equation, $\alpha$ is a Boltzmann parameter in [0,1]. It determines the speed of reducing in temperature and whether to accept new solution based on the Metropolis criterion.

$$
P t=\exp (\Delta E / T t)(4-4)
$$

In the equation, $\Delta \mathrm{E}$ is the difference between the current solution and current optimal solution.If $\Delta \mathrm{E}>0$, it shows current solution is superior to current optimal solution, and accept current solution. If $\Delta \mathrm{E}<0$, it shows current solution is inferior to current optimal solution, then accept current inferior solution with probability Pt. Thus, it can breakthrough local optimal solution of the algorithm and close in on the global optimal solution.

(5) Confirm stopping criterion. To make the algorithm more efficient and convenient, assume that ending algorithm of optimal solution is found when there are no change in optimal solution of several generations or iteration times is reached.

\section{Simulation and experiment}

Due to the strong nonlinear, discreteness and variable-structure of DC/DC converters, it is difficult for mathematical model with normal transfer function to describe all the characteristics of the system precisely. Simulink is one of the most important components of MATLAB. It provides a graphical programming environment for multi-domain dynamic systematic modeling, simulation and comprehensive analysis. In this paper, a simulated model of Buck circuit and system block diagram of control algorithm was built on Simulink. In this simulation, the circuit parameters are as follows: VIN $=30$ volt, VOUT $=12$ volt, applying different control strategies to SAGA algorithm, the population size is 200 , chromosome length is 16 , crossover rate is 0.86 ,mutation probability is 0.005 , the max-epoch is 200 , initial temperature is $25^{\circ} \mathrm{C}$, annealing factor is 0.9 . Let the range of e and ec be the fuzzy domain [-1,1], the time of simulation is 2 msec. The simulation achieved the best after 134 iterations, and we obtained $\mathrm{Ke}=0.0055, \mathrm{Kec}=0.012$. Results of the simulation is shown in Fig.5. 


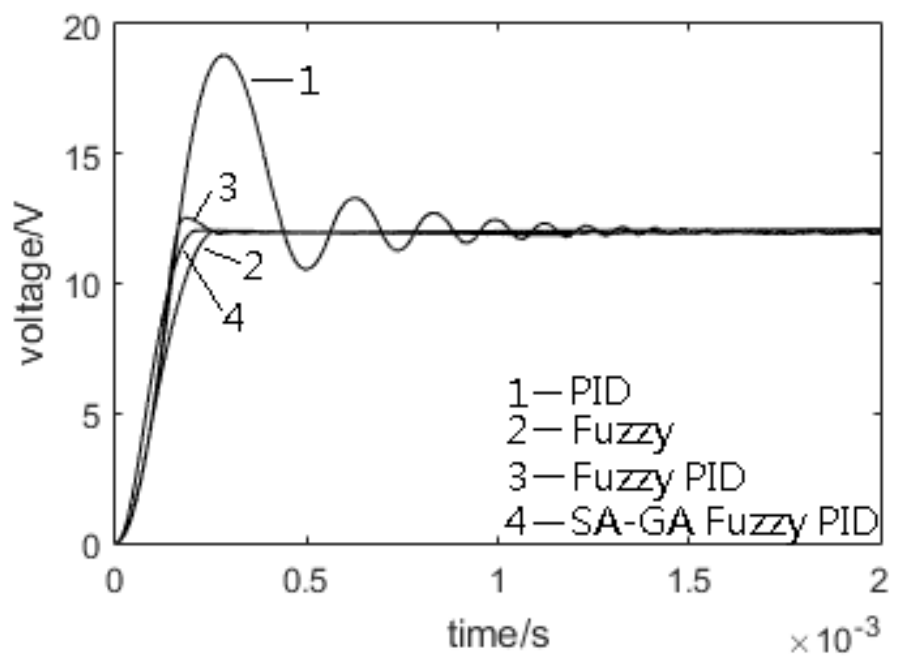

Fig. 5 The simulation results of different control method

It is shown in the output response curve of simulation, among different control strategies, linear PID cannot achieve good performance for the nonlinear system, the overshoot is large and the regulating time is long. Fuzzy control algorithm has empirical instructions of experts, it can improve the performance of system greatly. The overshoot is very small and the system response fast but with poor stability. Fuzzy PID has advantages of the two algorithm, which is small overshoot, quick response and stability. SAGA can regulate the parameter quickly with fast response, and track the signals of system rapidly. So fuzzy PID based on SAGA has rapid tracking performance, short adjusting time, strong robustness as well as little overshoot. From results above, it can be concluded that the proposedfuzzy PID based on SAGA gives good performance for DC/DC converters.

\section{Conclusion}

In this paper, due to the strong nonlinear and difficulty in control of DC/DC converters, a fuzzy PID control algorithm for DC/DC converters based on SAGA was proposed. It can adjust the parameters of PID and optimize them in real time. Simulation on MATLAB showed that the proposed approach improves the performance of system in terms of small overshoot and short adjusting time. SAGA is better than PID, Fuzzy PID algorithm. It should be noted that fuzzy PID controller based on SAGA has an excellent application future for DC/DC converters.

\section{Acknowledgments}

Author to whom correspondence should be addressed.Electronic mail:qyzhang@whut.edu.cn

\section{References}

[1]Jialiang Lu,Guanrong Chen, Hao Ying. Predictive fuzzy PID control: theory, design and simulation [J]. Inf. Sci.,2001,137:.

[2]Zhengxi Li, Me Liu, Dehui Sun, and Rentao Zhao. A complex control system based on the fuzzy PID control and state predictor feedback control[J]. Journal of University of Science and Technology Beijing(English Edition),2004,01:90-93.

[3]Zhi Yang,Jing Gao,Self-adjusting PID based on heredity arithmetic. Control and Decision .2000

[4]Mingran Li.Application of GA Fuzzy self-tuning PID controller based on magnetic levitation suspension system[D].Central South University,2012. 
[5]Fu-Cai Liu,Li-Huan Liang,Juan-Juan Gao. Fuzzy PID Control of Space Manipulator for Both Ground Alignment and Space Applications[J]. International Journal of Automation \& Computing,2014,04:353-360.

[6]LEI Lin, WANG Houjun. Adaptive Fuzzy PID Control Method Based on Identification Structure[J]. International Journal of Systems and Control,2006,01:15-21.

[7]Hu-min YAN,Yun-tao LEI,Dang-feng,PANG,Xiao HAN. Research of Speed Control System for DC Motor Based on Fuzzy PID[A]. IEEE Beijing Section.Proceedings of 2014 International Conference on Industrial Engineering and Information Technology[C].IEEE Beijing Section:,2014:5.

[8]Wang Shuyan,Shi Yu,Feng Zhongxu. A Method for Controlling a loading System Based on a Fuzzy PID Controller[J]. Mechanical Science and Technology,2011,01:166-172.

[9]Yonggang Peng,Xiaoping Luo,Wei Wei. A new fuzzy adaptive simulated annealing genetic algorithm and its convergence analysis and convergence rate estimation[J]. International Journal of Control, Automation and Systems,2014,123:.

[10]Chen Xueguo,Zhao Ruihuan,Ni Jianyi,Zou Hanfa. [Application of simulated annealing genetic algorithm in optimization of linear gradient elution conditions in HPLC].[J]. Sepu,2005,221:.

[11]Paul K. Bergey,Cliff T. Ragsdale,Mangesh Hoskote. A Simulated Annealing Genetic Algorithm for the Electrical Power Districting Problem.[J]. Annals OR,2003,121:.

[12]Dong-Kurl Kwak,Seung-Ho Lee,Do-Young Jung. A New Buck-Boost DC/DC Converter of High Efficiency by Soft Switching Technique[A]. China Electrotechnical society、 IEEE Power Electronics Society、National Science Foundation of China.2009 IEEE 6 (th) International Power Electronics and Motion Control Conference-ECCE Asia Conference Digests[C].China Electrotechnical society 、IEEE Power Electronics Society National Science Foundation of China:,2009:5.

[13]Li Qiao Wu Jie. Nonlinear PID Control Method Applied to DC-DC Converters[J]. Journal of South China University of Technology(Natural Science),2004,05:17-21.

[14]KUANG Jingguo,LIU Yue,ZHANG Renhong. Simulation and Research of DC-DC Converters Based on Novel Fuzzy-PID Control [J]. Modern Machinery,2012,04:66-70.

[15]SANG Hui-hui,YANG Yi,SHEN Cai-lin. Design of Simulation System for Buck Converter Based on PID Control[J]. Journal of Nantong University(Natural Science Edition), 2011,01:24-28.

[16] JING Li-xue,SU Hong-sheng,XIE Ming-jun,RAN Peng-cheng. Study of control strategies of voltage source BUCK converter[J]. Electric Drive Automation,2011,04:20-23. 